\title{
Coloured-fleshed potatoes after boiling: Promising sources of known antioxidant compounds
}

\author{
Maria Bellumori ${ }^{\mathrm{a}, \mathrm{b}}$, Marzia Innocenti ${ }^{\mathrm{a}, \mathrm{b}}$, Marco Michelozzi $^{\mathrm{d}}$, Lorenzo Cerretani ${ }^{\mathrm{c}}$, \\ Nadia Mulinacci ${ }^{\mathrm{a}, \mathrm{b}, *}$ \\ a Department of NEUROFARBA, Division of Pharmaceutical and Nutraceutical Sciences, via U. Schiff 6, 50019, Sesto F.no, Florence, Italy \\ ${ }^{\mathrm{b}}$ CeRA (Multidisciplinary Centre of Research on Food Sciences), Italy \\ c Pizzoli S.p.A., via Zenzalino Nord 1, 40054, Budrio, Bologna, Italy \\ d Istituto Bioscienze e BioRisorse, CNR, via Madonna del Piano 10, 50019, Sesto F.no, Florence, Italy
}

\section{A R T I C L E I N F O}

\section{Article history:}

Received 28 November 2016

Received in revised form 7 February 2017

Accepted 8 February 2017

Available online 10 February 2017

\section{Keywords:}

Solanum tuberosum

Anthocyanins

Phenols

HPLC/DAD/MS

Boiling

Antioxidant activity

Food analysis

Food composition

\begin{abstract}
A B S T R A C T
This study aims at evaluating the effect of boiling on the anthocyanin and phenolic acid content of varieties of yellow, pink and violet flesh colored potatoes grown in central Italy. The total phenolic acid content ranged from 33.4 to $1130 \mathrm{mg} / \mathrm{kg}$ of fresh weight (FW) in uncooked tubers and from 111 to $1375 \mathrm{mg} / \mathrm{kg}$ FW in cooked potatoes, with increments obtained for six varieties (Mz012, Mz064, Vitelotte Noir, Mz032, Mz080, Mz046) after boiling. The anthocyanin content decreased in a variety-dependent mode, but an increment was evidenced after cooking in one sample Mz012. A strong correlation was observed between antioxidant capacity and total anthocyanins for pink and violet-fleshed potatoes, suggesting that these compounds are mostly responsible of the antioxidant capacity of these tubers after boiling. Overall, the yellow-fleshed cultivars have shown lower antioxidant capacity (about 3.25 times lower) than the violet-fleshed tubers. Violet Mz064 variety registered the highest anthocyanin level and a daily intake of $100 \mathrm{~g}$ of this tuber contains up to $200 \mathrm{mg}$ of total phenols. Flesh-colored potatoes can represent an additional source of bioactive compounds, particularly of acylated anthocyanins in the human diet.
\end{abstract}

(C) 2017 Elsevier Inc. All rights reserved.

\section{Introduction}

The potato (Solanum tuberosum L.) is a staple food in many regions of the world and it is characterized by high carbohydrate, protein and vitamin $C$ contents. Freshly harvested potatoes contain about $20 \%$ dry matter from which the starch is about $60-80 \%$.In addition, the potato is low in fat and it is a good source of vitamins $\mathrm{B}_{1}, \mathrm{~B}_{3}, \mathrm{~B}_{6}$, folate, pantothenic acid, riboflavin and minerals, such as potassium, phosphorus and magnesium (Prokop and Albert, 2008).

The main phytochemicals of this tuber are solanine alkaloids, phenolic acids, anthocyanins and carotenoids; most of them are commonly in higher amount in peel (Ezekiel et al., 2013). They have attracted increasing attention in recent years due to their

\footnotetext{
* Corresponding author at: Department of NEUROFARBA, Division of Pharmaceutical and Nutraceutical Sciences, University of Florence, via U. Schiff 6, 50019, Sesto F.no, Italy.

E-mail address: nadia.mulinacci@unifi.it (N. Mulinacci).
}

health benefits; numerous studies have revealed a negative correlation between the intake of phytochemicals and chronic inflammation, cardiovascular diseases, cancer and diabetes (Acosta-Estrada et al., 2014; González-Castejón and RodriguezCasado, 2011). It is well known that phenolic compounds have antioxidant properties, protecting cellular constituents against oxidative damage and limiting the harmful effects of oxidative stress. Moreover, a recent study reported that purple potatoes acted as hypotensive agents, and were able to lower the risk of heart disease and stroke in hypertensive subjects without weight gain (Vinson et al., 2012). Choi et al. (2013) observed that a 20\% purple-fleshed potato powder intake improved both diabetes and lipid control in diabetic rats, by significantly improving serum insulin levels and lowering blood glucose and serum cholesterol levels. Furthermore, it was hypothesized that polyphenols from pigmented potatoes would decrease oxidative stress and inflammation in humans (Kaspar et al., 2011). Nowadays, the interest toward colored-fleshed potatoes is growing also because these tubers are richer in phenolic compounds. 
Potatoes are generally cooked before consumption, and the different cooking methods are important factors affecting not only the chemical composition and physical structure of the potato, but also the intake of bioactive compounds under normal dietary conditions (Tian et al., 2016). Changes in potato chemical composition mainly occur during storage and cooking and vary depending on the cultivar and growing conditions (Blessington et al., 2010; Brown et al., 2008; Burmeister et al., 2011; Ieri et al., 2011; Lachman et al., 2012).

This study aims to evaluate the anthocyanin and phenolic acid content in several varieties of potato with yellow, pink and violet coloured flesh selected by an Italian producer farm. The effect of boiling on the whole potato was evaluated by studying changes in the concentration of chlorogenic acid, its isomers and total anthocyanins before and after cooking. The antioxidant capacity was also estimated in the processed tubers using the ABTS assay. The final aim is to valorise the pigmented-fleshed potatoes which are not yet widely consumed in the Italian market.

\section{Material and methods}

\subsection{Materials}

The fresh tubers were obtained from breeding lines cultivated in the same way in a conventional system in the same field in the agricultural district of Bologna (Italy). Three yellow- (Mz032, Mz080, Mz046), three violet- (Mz128, Mz064, Vitelotte Noir) and two pink-fleshed (Mz011, Mz012) varieties of potatoes were studied. The tubers were kindly provided by Pizzoli S.p.A. (Bologna-Italy), both fresh and after boiling. A summary of the morphological characteristics and the common uses of all the varieties is reported in Table 1.

Ascorbic acid (99\% purity grade) was purchased from SigmaAldrich.

\subsection{Cooking process}

A representative amount of the whole tuber (about $4 \mathrm{~kg}$ for each variety) was boiled in a covered stainless steel pot on a moderate flame. The whole potatoes were boiled in water with $\mathrm{NaCl}(1 \%)$ and ascorbic acid for $15 \mathrm{~min}$. The ratio potatoes/water was $1: 4 \mathrm{w} / \mathrm{v}$. At the end of cooking, the tubers were peeled and frozen and these were used for the extraction of phenolic compounds.

\subsection{Sample preparation}

The potatoes of each variety $(1 \mathrm{~g})$ were extracted at room temperature, by stirring twice with $30 \mathrm{~mL}$ of $70 \% \mathrm{EtOH}$ adjusted to $\mathrm{pH} 2.0$ by $\mathrm{HCOOH}$, as already described in our previous study (Mulinacci et al., 2008). The hydroalcoholic solutions were analyzed by HPLC/DAD/MS, according to our previous work (Ieri et al., 2011) and used for the ability to scavenge the ABTS radical cation.

\subsection{HPLC/DAD/MS analysis}

Analysis was firstly carried out using a HP-1100 liquid chromatograph equipped with a DAD detector and a HP1100 MSD API-electrospray (Agilent-Technologies, Palo Alto, CA) operating in positive and negative ionization mode under the following conditions: gas temperature $350^{\circ} \mathrm{C}$, nitrogen flow rate $10 \mathrm{~L} / \mathrm{min}$, nebulizer pressure $35 \mathrm{psi}$, quadrupole temperature $30^{\circ} \mathrm{C}$, capillary voltage $4000 \mathrm{~V}$, and applied fragmentors in the range $50-250 \mathrm{~V}$.

Further identification and characterization of the phenolic compounds was performed by a TOF-MS with an ESI interface (Agilent Technologies) under the following conditions: gas temperature $300^{\circ} \mathrm{C}$, nitrogen flow rate $12 \mathrm{~L} / \mathrm{min}$, nebulizer pressure $20 \mathrm{psi}$, capillary voltage $3800 \mathrm{~V}$, and applied fragmentors in the range $80-300 \mathrm{~V}$.

The column was a Synergi Max RP $80 \mathrm{~A}(4 \mu \mathrm{m} ; 150 \mathrm{~mm} \times 3 \mathrm{~mm}$ i.d.) from Phenomenex (Castel-Maggiore, BO, Italy). The mobile phases were: (A) acidified water ( $\mathrm{pH} 2.0)$ and (B) acetonitrile. The following multistep linear gradient was applied: from $95 \%$ to $78 \% \mathbf{A}$ in $8 \mathrm{~min}, 4 \mathrm{~min}$ to reach $74 \% \mathbf{A}$, then $13 \mathrm{~min}$ to arrive at $65 \% \mathbf{A}$, and finally 3 min to reach $100 \%$ B with a final plateau of $4 \mathrm{~min}$. The total time of analysis was $32 \mathrm{~min}$, flow rate was $0.4 \mathrm{~mL} / \mathrm{min}$, and oven temperature was $26 \pm 0.5^{\circ} \mathrm{C}$, as described in our previous study (Ieri et al., 2011).

\subsection{Quantitative determination of phenolic acids and anthocyanins}

The phenolic acids were evaluated by HPLC/DAD using a sixpoint calibration curve of chlorogenic acid (3-caffeoylquinic acidpurity $\geq 99 \%$ ) (Extrasynthèse, Genay, France) at $330 \mathrm{~nm}$ $\left(r^{2}=0.999\right)$, while the anthocyanin content was determined by HPLC/DAD using a five-point calibration curve of malvin chloride (MW 691 from Extrasynthèse; purity $\geq 95 \%$ ) at $520 \mathrm{~nm}\left(r^{2}=0.999\right)$.

\subsection{Antioxidant capacity by the 2,2'-azino-bis(3- ethylbenzothiazoline-6-sulphonate) (ABTS) method}

The ABTS method (Miller et al., 1996) was used for the determination of antioxidant capacity. The hydroalcoholic extracts were diluted with distilled water (100 times) and immediately subjected to reaction with the radicals; absorbance was measured after the addition of a $200 \mu \mathrm{L}$ sample to $2 \mathrm{~mL}$ of radical solution after 2 min. For the blank experiment, $200 \mu \mathrm{L}$ of water was added. The antioxidant activity was calculated as follows by the equation:

$\%$ inibition $\mathrm{ABTS}=\left[\left(A_{0}-A_{\mathrm{t}}\right) / A_{0}\right] \times 100$

In agreement with this study, $54.8 \mathrm{mg}$ ABTS (Sigma-Aldrich, Milan, Italy) was dissolved in $20 \mathrm{~mL}$ phosphate buffer ( $\mathrm{pH} 7.0$; $5 \mathrm{mM}$ ) and activated to $\mathrm{ABTS}^{\bullet+}$ radical by adding $1 \mathrm{~g}$ of $\mathrm{MnO}_{2}$ (activation time $20 \mathrm{~min}$ ). Then the solution was centrifuged, filtered and diluted with the buffer solution to obtain $A_{734}\left(t_{0}\right)=$ $0.800 \pm 0.02 \mathrm{~nm}$. Absorbance of the solution was measured at a wavelength of $734 \mathrm{~nm}$. Values are the means of three repetitions.

Table 1

Morphological characteristics of the analyzed varieties.

\begin{tabular}{|c|c|c|c|c|c|c|c|}
\hline variety or code & country of origin & maturity & skin colour & flesh colour & tuber form & $\%$ dry matter & cooking use \\
\hline Mz011 & Italy & late & pink & pink & long oval & $16-19$ & boiled, mashed \\
\hline Mz012 & Netherland & medium late & dark pink/red & dark pink/red & long oval & 17 & boiled, mashed \\
\hline Mz128 & Netherland & late & brown/violet & light violet & round oval & $16-18$ & boiled, mashed \\
\hline Mz064 & Netherland & medium late & brown/violet & blue/violet & small long & $20-23$ & boiled, mashed and chips \\
\hline Vitelotte Noir & France & very late & dark violet & dark violet & long irregular & 23 & boiled, mashed and chips \\
\hline Mz032 & United Kingdom & medium early & dark yellow and purple & dark yellow & small long & $>23$ & boiled, mashed \\
\hline $\mathrm{Mz046}$ & United Kingdom & medium early & dark yellow & dark yellow & small long & $>23$ & boiled, mashed \\
\hline Mz080 & Netherland & medium late & yellow with red eyes & yellow & oval & $18-22$ & multiuse \\
\hline
\end{tabular}




\subsection{Statistical analysis}

Data were not normally distributed (Kolmogorov-Smirnov one sample test) and were analyzed by the non-parametric KruskalWallis ANOVA, followed by the Mann-Whitney $U$ test for multiple comparisons using SYSTAT 12.0 software (Systat Software Inc., Richmond, CA). Differences were accepted as significant at the 5\% level.

\section{Results and discussion}

\subsection{Phenolic acid and anthocyanin content}

The chromatographic profiles obtained according to our previous works (Ieri et al., 2011; Mulinacci et al., 2008), demonstrated the same compounds in both raw and cooked potatoes, (Tables 2 and 3). The same phenolic acid pattern was highlighted, and the main phenolic acids detected were 3caffeoylquinic, 5-caffeoylquinic, 4-caffeoylquinic and ferulic acids. The identified anthocyanins were acylated glycosides of pelargonidin, malvidin, petunidin, peonidin and delphinidin; the rutinosides in C3 were the dominant forms, acylated with a cinnamoyl residue in $\mathrm{C} 4$ of the rhamnose unit.

For all of the varieties, the total levels of anthocyanins and phenolic acids varied significantly between raw and cooked tubers, as shown in Tables 2 and 3. Violet potatoes were rich in anthocyanins, and the Mz064 cultivar in particular registered the highest levels (691 mg/kg FW; Fig. 1a). The boiling was responsible of a significant decrease in the amount of anthocyanins when compared to raw potatoes (Fig. 1a) with the highest loss showed for Mz128 and Mz064 (66.1\% and 36.7\% respectively). In only one case, for Mz012 cultivar, a statistically significant increase in the amount of total anthocyanins was observed after cooking, with an increment of more than a double (from 88.9 in fresh to $182 \mathrm{mg} / \mathrm{kg}$ in cooked potato).

Contradictory results were found in the literature relating to the effect of cooking on the polyphenol content of potatoes. Our data are partly in accordance with the study performed by Brown et al. that reported a significant decrease in the total anthocyanins in five pigmented varieties after boiling, microwaving and baking (Brown et al., 2008). According to Lachman et al., the increase in anthocyanin content after cooking is strongly variety-dependent (Lachman et al., 2013). Various factors influence the composition of anthocyanins in fresh tubers, such as the cultivar and climatic conditions including altitude and storage conditions (Lachman et al., 2012).

As reported in Table 2, a significant decrease in the level of all the anthocyanins was detected between raw and cooked potatoes, particularly for the varieties Mz011, Mz064 and Vitelotte Noir. In the variety Mz128, the amount of malvidin 3-O-p-coum-rut-5-Oglu did not vary significantly after cooking, while the statistical Mann-Whitney $U$ test showed significant variations for all of the other anthocyanins present. Significant increases were shown in the Mz012 variety for pelargonidin 3-O-rut-5-O-glu, pelargonidin 3-O-rut, pelargonidin derivative 1 , pelargonidin 3-O-caf-rut-5-Oglu, pelargonidin 3-O-cis-p-coum-rut-5-O-glu, pelargonidinderivative 2 and pelargonidin 3-O-p-coum-rut-5-O-Glu. Pelargonidin 3-O-p-coum-rut-5-O-glu was the major anthocyanin in the pink varieties (Mz011, Mz012) and in Vitelotte Noir; the same anthocyanin pattern was found in these three varieties. The violet varieties Mz128 and Mz064 showed high concentrations of petunidin 3-O-p-coum-rut-5-O-Glu.

The phenolic acid content was determined for the eight varieties, and ranged from 33.4 (Mz080) to $1130 \mathrm{mg} / \mathrm{kg}$ FW (Vitelotte Noir) in fresh potatoes, and from 111 (Mz080) to $1375 \mathrm{mg} / \mathrm{kg} \mathrm{FW}$ (Mz012) in cooked potatoes (Table 3). In the $\mid$

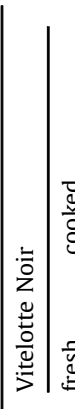

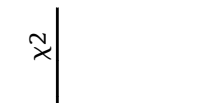

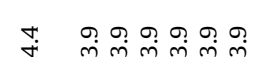

营 $\frac{00}{00}$

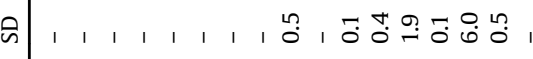

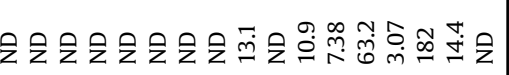

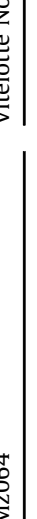

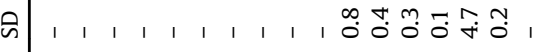

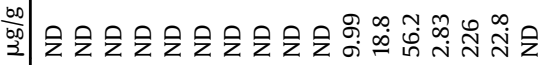

×

********

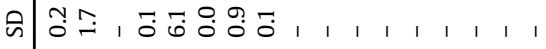

范

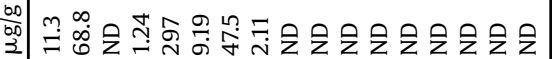

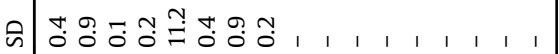

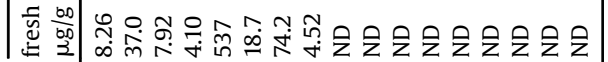

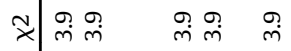

** ** * $\tilde{z}$ *

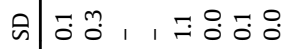

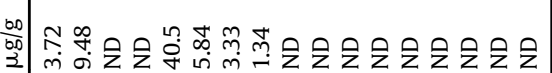

$\stackrel{\infty}{\sim}$

ง

$\dot{0}$

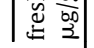

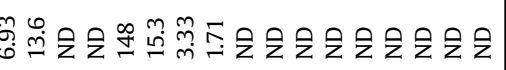

$$
\text { भे サं サ্ণ }
$$

*n.

$\sim \tilde{z}$

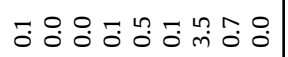

की

密。

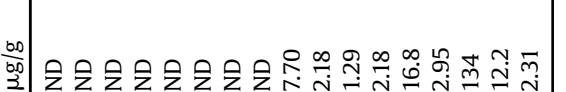

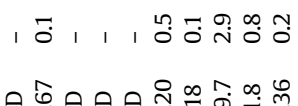

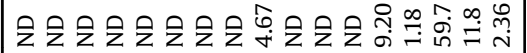

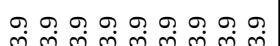

*********

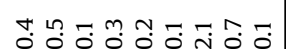

$1,1,1,1$

0

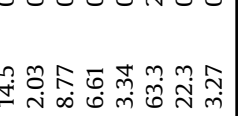

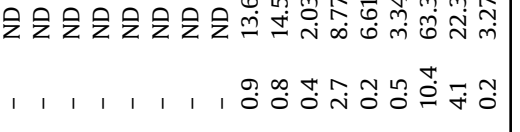

ค

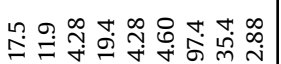

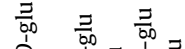

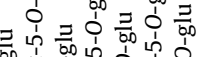

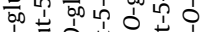

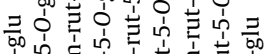

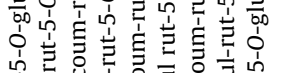




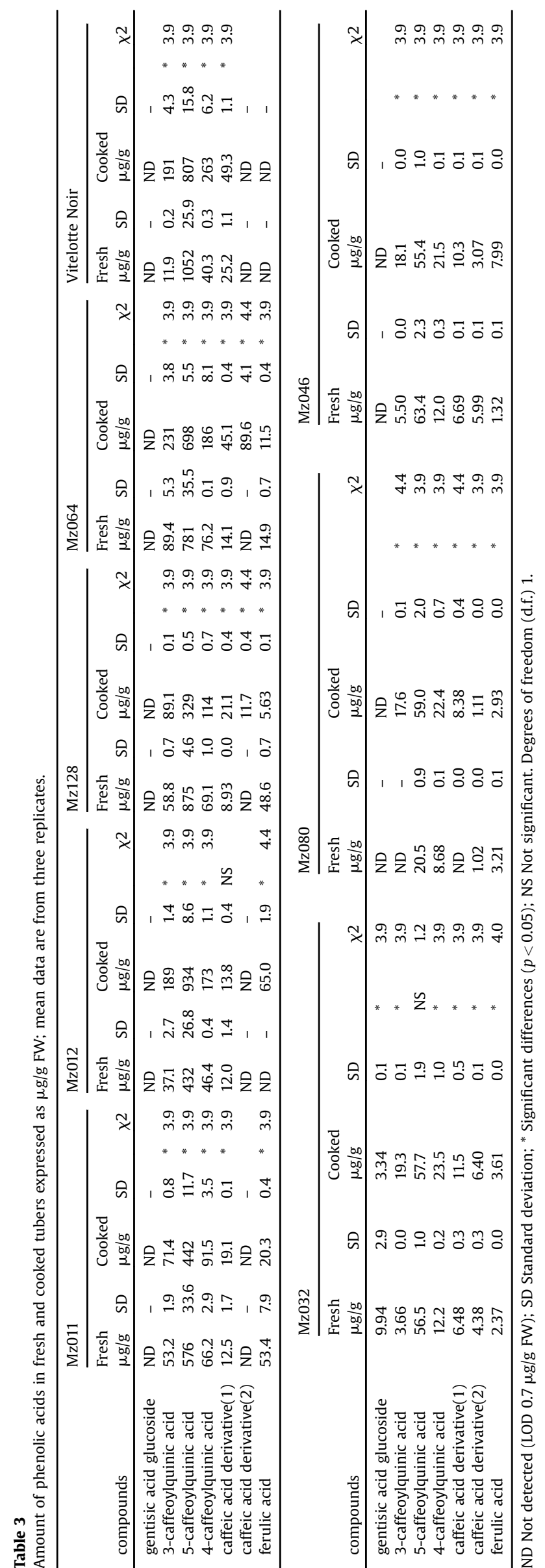

yellow-fleshed varieties the average total phenolic acid content values ranged from 33.4 to $94.9 \mathrm{mg} / \mathrm{kg} \mathrm{FW}$, with changes in their content less evident after cooking (from 111 to $125 \mu \mathrm{g} / \mathrm{g}$ FW). However, according to our previous works (Ieri et al., 2011; Mulinacci et al., 2008), the total amount of these phenols in the yellow-flesh varieties is much lower in comparison to the content observed in the red and violet varieties (Fig. 1). A recent study reported that the phenolic acids were from three to four times higher in purple- and red-fleshed potatoes than in whitefleshed tubers (Ezekiel et al., 2013). An analogous result was obatained for our samples, although with a greater difference (about ten times).

The cooking technique was responsible for a significant increase in the amount of phenolic acids for all of the yellow-fleshed varieties, and the content of these compounds increased in relation to the variety. As shown in Fig. 1b, the highest difference between raw and cooked tubers was observed for the variety Mz080. After cooking, a significant increase in the total amount of phenolic acids was also shown in violet and pink fleshed potatoes (Mz064, Vitelotte Noir and Mz012); the highest increase (2.6 times) was observed for the variety Mz012. These results were in agreement with literature data showing greater levels of phenolic acids in cooked than in raw potatoes (Blessington et al., 2010; Burgos et al., 2013). Faller and Fialho (2009) compared the total phenolic content after boiling, steaming and microwaving and found that the phenolic content increased by $81.4 \%, 22.8 \%$ and $80.81 \%$, respectively. It has been demonstrated that cooking interferes with the level of phenolic compounds by inducing their release and making them more available for extraction (Burgos et al., 2013). Cooking may also facilitate the extractability of polyphenols by altering the matrix, resulting in higher recoveries and inactivating enzymes that otherwise oxidize these compounds (Navarre et al., 2010).

Lachman et al. reported that the total level of phenolics, chlorogenic acids, flavonols and vitamin $\mathrm{C}$ did not significantly decrease after cooking by microwaving, baking, boiling, steaming or stir-frying methods (Lachman et al., 2012); this behaviour could be attributed to the disruption of plant cell walls providing a better extractability and to the breakage of chemical bonds of higher molecular weight polyphenols, forming soluble low molecular weight molecules.

In contrast, the amount of phenolic acids found in cooked tubers from the Mz011 and Mz128 varieties were significantly lower than in raw samples, in agreement with previous studies (Ezekiel et al., 2013; Lemos et al., 2015). It was reported that cooking had a strong detrimental effect on the total levels of phenolics; the total phenolics in uncooked potatoes were $209 \pm 36 \mathrm{mg}$ GAE$/ 100 \mathrm{~g}$ FW, while baking decreased the value to $38.1 \pm 8 \mathrm{mg} \mathrm{GAE} / 100 \mathrm{~g}$, boiling decreased it to $138 \pm 4 \mathrm{mg}$ $\mathrm{GAE} / 100 \mathrm{~g}$, microwaving decreased it to $74.0 \pm 1 \mathrm{mg} \mathrm{GAE} / 100 \mathrm{~g}$, and steaming decreased it to $130 \pm 4 \mathrm{mg} \mathrm{GAE} / 100 \mathrm{~g}$ (Lemos et al., 2015).

Nevertheless, these strong reductions were mainly observed in potatoes not cooked as whole tubers. Consequently, concomitant factors induced the decrements: the browning phenomenon during slicing, the reduced pulp surface exposed to hot water, the percentage of water absorbed by the starch during cooking and most importantly the presence of peel (Mulinacci et al., 2008), because it is well known that potato peel contains high quantities of phenolic compounds. In 1989 Mondy and Gosselin found that potatoes cooked with peel had a greater amount of total phenols in the cortex and internal tissues, and this has been attributed to the migration of phenolics from the peel towards the inside of the tuber (Ezekiel et al., 2013).

As reported in Table 3, the major phenolic compound in all the varieties (5-caffeoylquinic acid), was significantly reduced after 
a
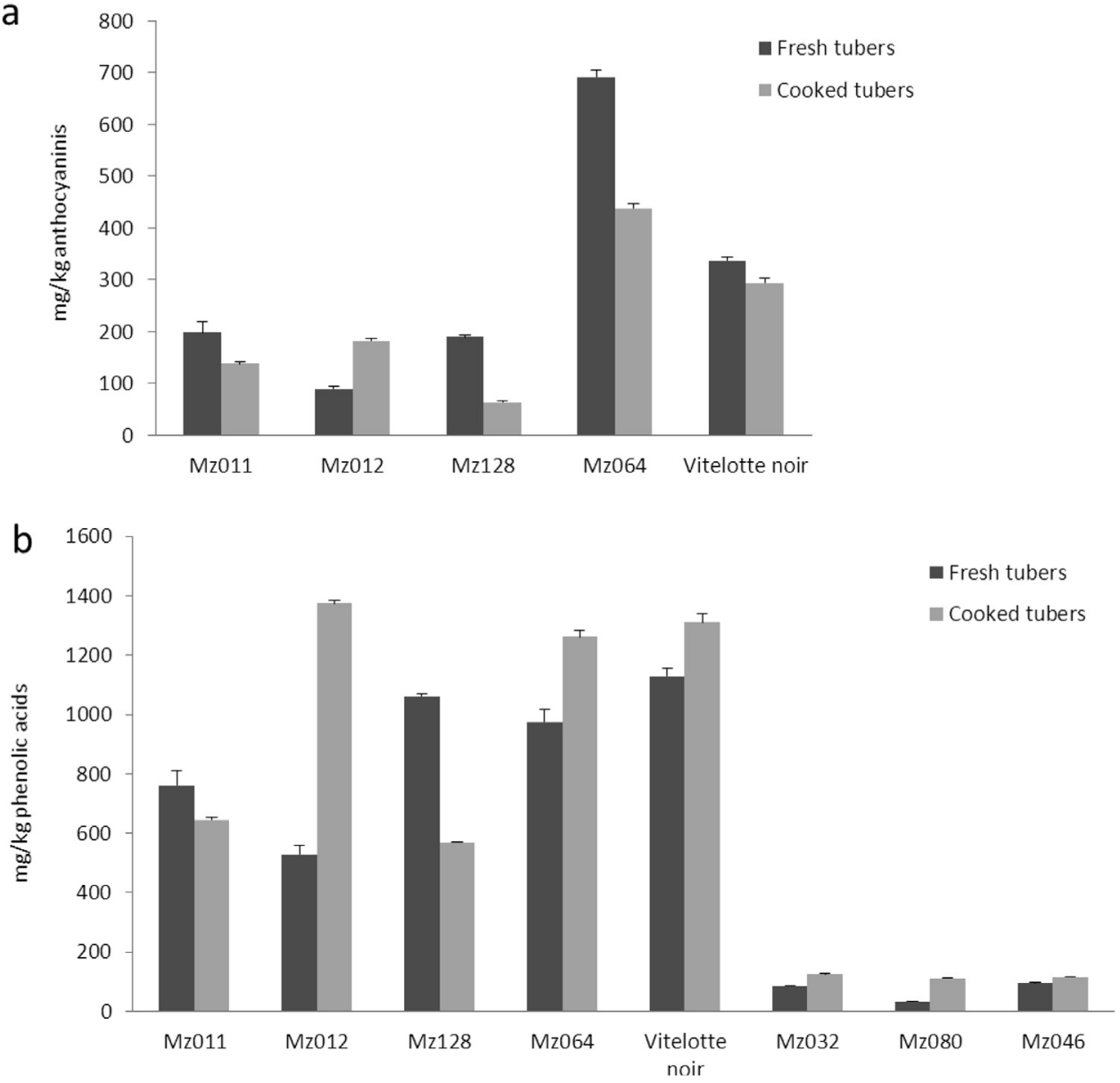

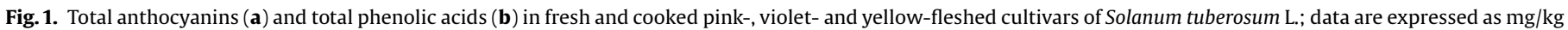
FW (fresh weight). The differences between fresh and cooked tubers are significant for all the varieties ( $\chi^{2} 3.9$; degrees of freedom (d.f.) 1).

cooking, except in the Mz011, Mz128, Mz064, Vitelotte Noir and Mz046 varieties. We can hypothesize a partial inter-conversion among the isomers in these varieties, particularly from 5-caffeoylquinic acid to 3-caffeoylquinic acid. According to the peculiarities of the different varieties, gentisic acid monoglucoside was identified and quantified only in the Mz032 sample.

\subsection{Antioxidant capacity}

Potatoes are a source of phenolic acids but also of anthocyanins and carotenoids, which can contribute to increase the antioxidant intake in the human diet (Ezekiel et al., 2013; Hamouz et al., 2011; Lachman and Hamouz, 2005; Reyes et al., 2005). Considering that

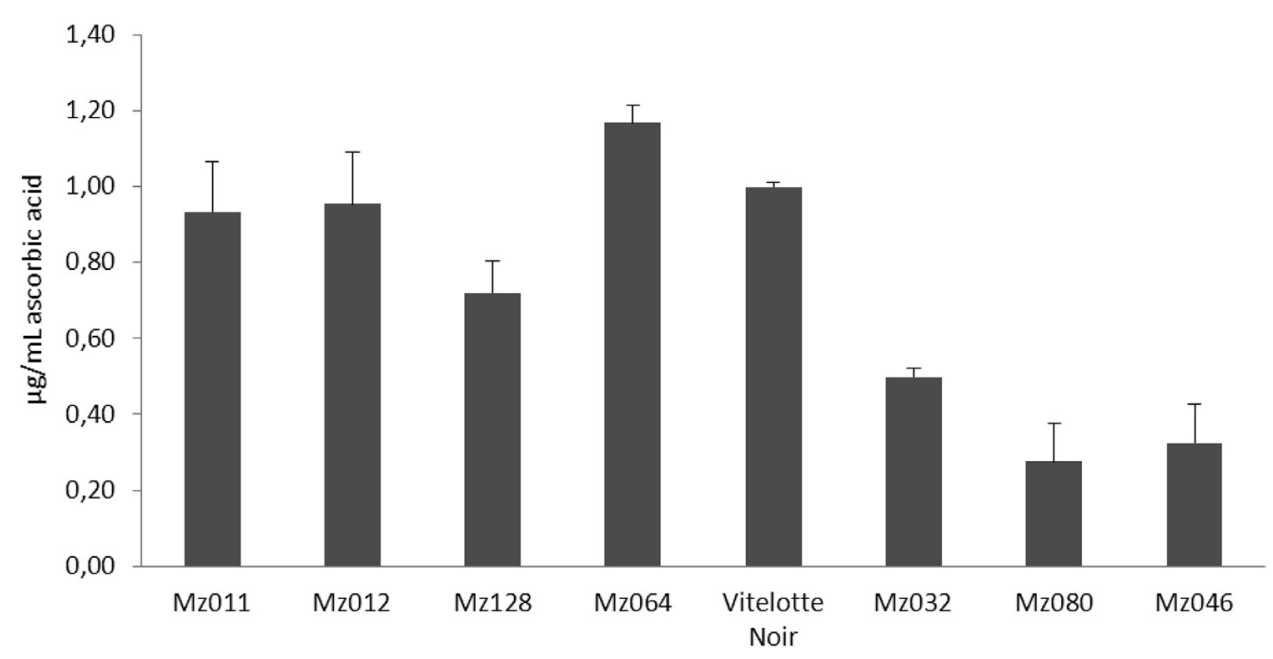

Fig. 2. Antioxidant capacity (expressed as $\mu \mathrm{g} / \mathrm{mL}$ ascorbic acid) measured for each cooked sample of potatoes. 
the human consumption of the tuber occurs after cooking, the antioxidant capacity of our samples was assayed by the ABTS method only after boiling. As reported in Fig. 2, the lowest antioxidant capacity was observed in the yellow fleshed varieties, averaging $0.37 \mu \mathrm{g} / \mathrm{mL}$ of ascorbic acid. In agreement with other studies (Külen et al., 2013; López-Cobo et al., 2014) that reported blue and purple potatoes possessing a higher antioxidant capacity compared to the white-yellow tubers, the blue-violet fleshed potato (Mz064) showed the highest antioxidant capacity in the ABTS assay.

The obtained correlation coefficients are very similar considering phenolic acids (Fig. 3a), anthocyanins (Fig. 3b) and the total phenols (Fig. 3c), with $r^{2}$ values from 0.83 to 0.89 . A better correlation $\left(r^{2}=0.98\right)$ was found between the antioxidant capacity and total phenolic acid amount in the yellow-fleshed cultivars selected. The pink and violet-fleshed potatoes did not show a correlation between antioxidant capacity and total phenolic acids $\left(r^{2}=0.5\right)$, even if they contain considerably higher levels of caffeoylquinic acid isomers than the yellow varieties (Ieri et al., 2011). As expected and according to previous results (Brown, 2005; Brown et al., 2005; Lachman et al., 2012, 2009), these pigmented

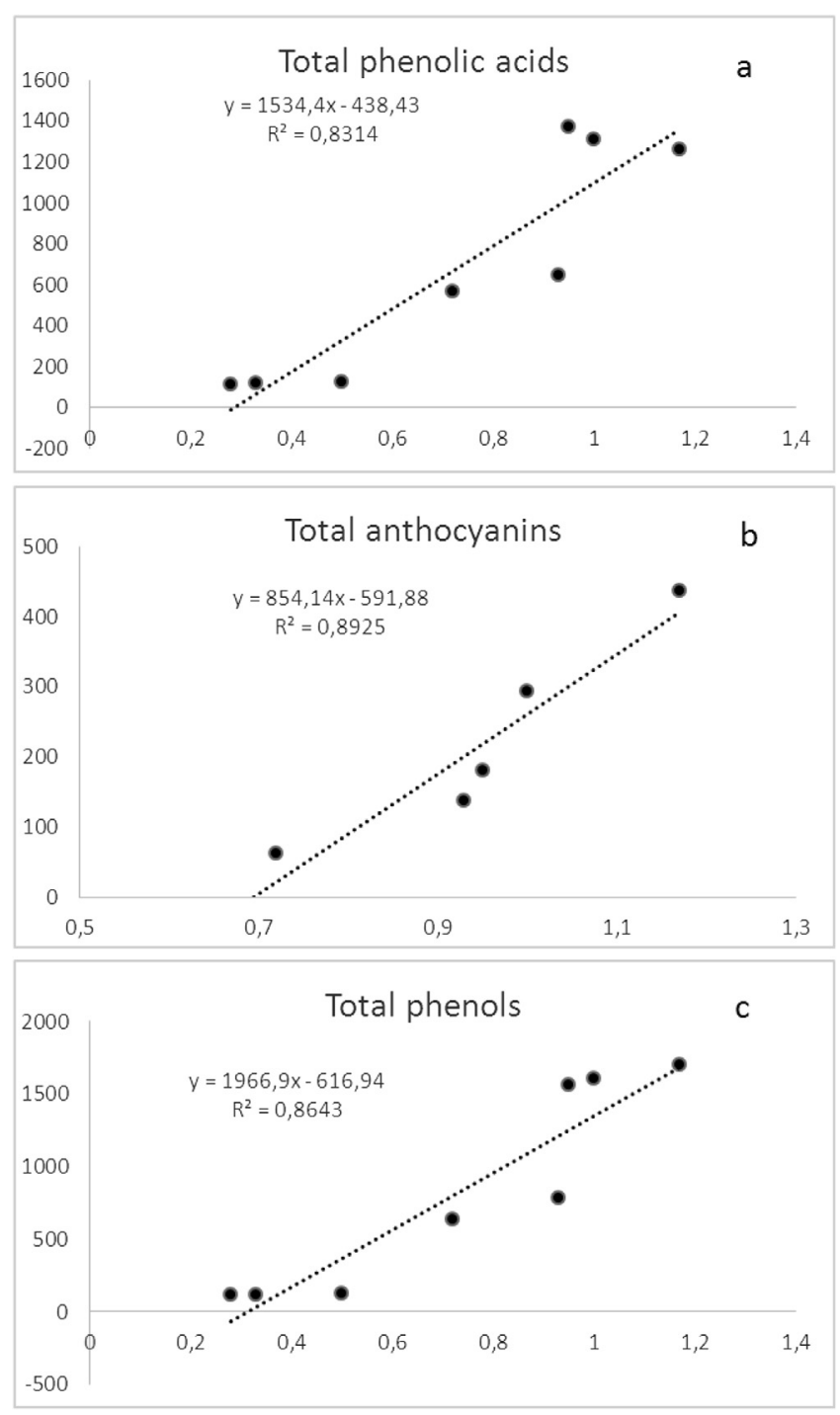

Fig. 3. Correlation between total phenolic acid (a), anthocyanin (b) and total phenol (c) contents expressed as $\mathrm{mg} / \mathrm{kg} \mathrm{FW}$ and antioxidant capacity expressed as $\mu \mathrm{g} / \mathrm{mL}$ ascorbic acid. tubers showed a higher and positive correlation between antioxidant capacity and total anthocyanins (Fig. 3b), with anthocyanins being mainly responsible for the antioxidant properties. The ORAC and FRAP assays revealed that antioxidant levels in red or purple-fleshed potatoes were two or three times higher than in white or yellow-fleshed potatoes (Teow et al., 2007). Overall, these varieties are a natural source of acylated anthocyanins and cinnamoyl acids and can positively contribute to increased antioxidant intake in the diet.

\section{Conclusions}

In this study, we investigated the effect of boiling on the total levels of phenolic compounds in different varieties cultivated in the same experimental conditions. Significant positive and negative variations were found in the concentration of phenolic acids and anthocyanins in the cooked tubers, highlighting a variety-dependent correlation. The antioxidant capacity estimated by the ABTS assay on the cooked tubers demonstrated a similar positive correlation with the content of phenolic acids, total anthocyanins and total phenols.

The boiled pink and violet-fleshed potatoes showed the highest efficacy as radical scavengers in the ABTS test. Chlorogenic acid provided the greatest contribution to antioxidant capacity in yellow fleshed potatoes, while anthocyanins were the major contributors as antioxidants in pink and violet-fleshed tubers. Our results indicate that pink and violet-fleshed potatoes show significantly higher antioxidant potency than yellow-fleshed varieties. Thus, the use of violet-fleshed potatoes in cuisine should be recommended because it can help to increase the daily intake of these interesting acylated anthocyanins.

\section{Acknowledgments}

This work was supported by the University of Florence and by Pizzoli S.p.A., Budrio (Bologna). We are grateful to Ente Cassa di Risparmio di Firenze for supplying part of the instrumentation used for this research.

\section{References}

Acosta-Estrada, B.A., Gutiérrez-Uribe, J.A., Serna-Saldívar, S.O., 2014. Bound phenolics in foods, a review. Food Chem. 152, 46-55. doi:http://dx.doi.org/ 10.1016/j.foodchem.2013.11.093.

Blessington, T., Nzaramba, M.N., Scheuring, D.C., Hale, A.L., Reddivari, L., Miller, J.C. 2010. Cooking methods and storage treatments of potato: effects on carotenoids, antioxidant activity, and phenolics. Am. J. Potato Res. 87, 479-491 doi:http://dx.doi.org/10.1007/s12230-010-9150-7.

Brown, C.R., Culley, D., Yang, C.-P., Durst, R., Wrolstad, R., 2005. Variation of anthocyanin and carotenoid contents and associated antioxidant values in potato breeding lines. J. Am. Soc. Hortic. Sci. 130, 174-180.

Brown, C.R., Durst, R.W., Wrolstad, R., De Jong, W., 2008. Variability of phytonutrient content of potato in relation to growing location and cooking method. Potato Res. 51, 259-270. doi:http://dx.doi.org/10.1007/s11540-008-9115-0.

Brown, C.R., 2005. Antioxidants in potato. Am. J. Potato Res. 82, 163-172. doi:http:// dx.doi.org/10.1007/BF02853654.

Burgos, G., Amoros, W., Muñoa, L., Sosa, P., Cayhualla, E., Sanchez, C., Díaz, C., Bonierbale, M., 2013. Total phenolic, total anthocyanin and phenolic acid concentrations and antioxidant activity of purple-fleshed potatoes as affected by boiling. J. Food Compos. Anal. 30, 6-12. doi:http://dx.doi.org/10.1016/j. jfca.2012.12.001.

Burmeister, A. Bondiek, S., Apel, L., Kühne, C., Hillebrand, S., Fleischmann, P., 2011. Comparison of carotenoid and anthocyanin profiles of raw and boiled Solanum tuberosum and Solanum phureja tubers. J. Food Compos. Anal. 24, 865-872. doi: http://dx.doi.org/10.1016/j.jfca.2011.03.006.

Choi, M.K., Park, S.J., Eom, S.H., Kang, M.H., 2013. Anti-diabetic and hypolipidemic effects of purple-fleshed potato in streptozotocin-induced diabetic rats. Food Sci. Biotechnol. 22, 1-6. doi:http://dx.doi.org/10.1007/s10068-013-0231-5.

Ezekiel, R., Singh, N., Sharma, S., Kaur, A., 2013. Beneficial phytochemicals in potato-a review. Food Res. Int. 50, 487-496. doi:http://dx.doi.org/10.1016/j. foodres.2011.04.025. 
Faller, A.L.K., Fialho, E., 2009. The antioxidant capacity and polyphenol content of organic and conventional retail vegetables after domestic cooking. Food Res. Int. 42, 210-215. doi:http://dx.doi.org/10.1016/j.foodres.2008.10.009.

González-Castejón, M., Rodriguez-Casado, A., 2011. Dietary phytochemicals and their potential effects on obesity: a review. Pharmacol. Res. 64, 438-455. doi: http://dx.doi.org/10.1016/j.phrs.2011.07.004.

Hamouz, K., Lachman, J., Pazderů, K., Tomášek, J., Hejtmánková, K., Pivec, V., 2011. Differences in anthocyanin content and antioxidant activity of potato tubers with different flesh colour. Plant Soil Environ. 57, 478-485.

Ieri, F., Innocenti, M., Andrenelli, L., Vecchio, V., Mulinacci, N., 2011. Rapid HPLC/ $\mathrm{DAD} / \mathrm{MS}$ method to determine phenolic acids, glycoalkaloids and anthocyanins in pigmented potatoes (Solanum tuberosum L.) and correlations with variety and geographical origin. Food Chem. 125, 750-759. doi:http://dx.doi.org/ 10.1016/j.foodchem.2010.09.009.

Külen, O., Stushnoff, C., Holm, D.G., 2013. Effect of cold storage on total phenolics content, antioxidant activity and vitamin C level of selected potato clones. J. Sci. Food Agric. 93, 2437-2444. doi:http://dx.doi.org/10.1002/jsfa.6053.

Kaspar, K.L., Park, J.S., Brown, C.R., Mathison, B.D., Navarre, D.A., Chew, B.P., 2011 Pigmented potato consumption alters oxidative stress and inflammatory damage in men. J. Nutr. 141, 108-111. doi:http://dx.doi.org/10.3945/ jn.110.128074.

López-Cobo, A., Gómez-Caravaca, A.M., Cerretani, L., Segura-Carretero, A. Fernández-Gutiérrez, A., 2014. Distribution of phenolic compounds and other polar compounds in the tuber of Solanum tuberosum L. by HPLC-DAD-q-TOF and study of their antioxidant activity. J. Food Compos. Anal. 36,1-11. doi:http:// dx.doi.org/10.1016/j.jfca.2014.04.009.

Lachman, J., Hamouz, K., 2005. Red and purple coloured potatoes as a significant antioxidant source in human nutrition-a review. Plant Soil Environ. 51, 477 482.

Lachman, J., Hamouz, K., Šulc, M., Orsák, M., Pivec, V., Hejtmánková, A., Dvořák, P., Čepl, J., 2009. Cultivar differences of total anthocyanins and anthocyanidins in red and purple-fleshed potatoes and their relation to antioxidant activity. Food Chem. 114, 836-843. doi:http://dx.doi.org/10.1016/j.foodchem.2008.10.029.

Lachman, J., Hamouz, K., Orsák, M., Pivec, V., Hejtmánková, K., Pazderů, K., Dvořák P., Čepl, J., 2012. Impact of selected factors-cultivar, storage, cooking and baking on the content of anthocyanins in coloured-flesh potatoes. Food Chem. 133, 1107-1116. doi:http://dx.doi.org/10.1016/j.foodchem.2011.07.077.
Lachman, J., Hamouz, K., Musilová, J., Hejtmánková, K., Kotíková, Z., Pazderù, K., Domkářová, J., Pivec, V., Cimr, J., 2013. Effect of peeling and three cooking methods on the content of selected phytochemicals in potato tubers with various colour of flesh. Food Chem. 138, 1189-1197. doi:http://dx.doi.org/ 10.1016/j.foodchem.2012.11.114.

Lemos, M.A., Aliyu, M.M., Hungerford, G., 2015. Influence of cooking on the levels of bioactive compounds in Purple Majesty potato observed via chemical and spectroscopic means. Food Chem. 173, 462-467. doi:http://dx.doi.org/10.1016/j. foodchem.2014.10.064.

Miller, N.J., Sampson, J., Candeias, L.P., Bramley, P.M., Rice-evans, C.A., 1996. Antioxidant activities of carotenes and xanthophylls. FEBS Lett. 384, 240-242.

Mulinacci, N., Ieri, F., Giaccherini, C., Innocenti, M., Andrenelli, L., Canova, G. Saracchi, M., Casiraghi, M.C., 2008. Effect of cooking on the anthocyanins, phenolic acids, glycoalkaloids, and resistant starch content in two pigmented cultivars of Solanum tuberosum L. J. Agric. Food Chem. 56, 11830-11837. doi: http://dx.doi.org/10.1021/jf801521e.

Navarre, D.A., Shakya, R., Holden, J., Kumar, S., 2010. The effect of different cooking methods on phenolics and vitamin C in developmentally young potato tubers. Am. J. Potato Res. 87, 350-359. doi:http://dx.doi.org/10.1007/s12230-010-91418.

Prokop, S., Albert, J., 2008. Potatoes, nutrition and diet. Food Agric. Organ. Factsheet $1-2$.

Reyes, L.F., Miller, J.C., Cisneros-Zevallos, L., 2005. Antioxidant capacity, anthocyanins and total phenolics in purple- and red-fleshed potato (Solanum tuberosum L.) genotypes. Am. J. Potato Res. 82, 271-277. doi:http://dx.doi.org/ $10.1007 /$ BF02871956.

Teow, C.C., Truong, V.-Den, McFeeters, R.F., Thompson, R.L., Pecota, K.V., Yencho, G. C., 2007. Antioxidant activities, phenolic and carotene contents of sweet potato genotypes with varying flesh colours. Food Chem. 103, 829-838. doi:http://dx. doi.org/10.1016/j.foodchem.2006.09.033.

Tian, J., Chen, J., Ye, X., Chen, S., 2016. Health benefits of the potato affected by domestic cooking: a review. Food Chem. 202, 165-175. doi:http://dx.doi.org/ 10.1016/j.foodchem.2016.01.120.

Vinson, J.A., Demkosky, C.A., Navarre, D.A., Smyda, M.A., 2012. High-antioxidant potatoes: acute in vivo antioxidant source and hypotensive agent in human after supplementation to hypertensive subjects. J. Agric. Food Chem. 60, 67496754. doi:http://dx.doi.org/10.1021/jf2045262. 